EPJ Web of Conferences 41, 05035 (2013)

DOI: $10.1051 /$ epjconf/20134105035

(C) Owned by the authors, published by EDP Sciences, 2013

\title{
Observation of Two-Exciton States in Perylene Bisimide Aggregates
}

\author{
S. Wolter ${ }^{1}$, M. Seidel ${ }^{1}$, F. Würthner ${ }^{2}$, and S. Lochbrunner ${ }^{1}$ \\ ${ }^{1}$ Institut für Physik, Universität Rostock, Universitätsplatz 3, 18051 Rostock, Germany \\ ${ }^{2}$ Institut für Organische Chemie and Röntgen Research Center for Complex Material Systems, \\ Universität Würzburg, Am Hubland, Würzburg, Germany
}

\begin{abstract}
The behavior of excitons on perylene bisimide aggregates is investigated at high excitation densities by femtosecond absorption spectroscopy. Indications for a significant population in the two-exciton manifold are found.
\end{abstract}

\section{Introduction}

In future, supramolecular organic structures will be most probably employed in a wide range of photonic devices like organic solar cells or light emitting diodes. Therefore, the behavior of their fundamental electronic excitations, so-called Frenkel excitons, attracts intense research activities. Promising building blocks for supramolecular structures are perylene bisimide (PBI1) J-aggregates, which are investigated in this contribution. The oscillator strength of their HOMO-LUMO-transition is large and they exhibit a high fluorescence yield [1]. Additionally, the exciton motion upon these aggregates is restricted to one dimension which provides an interesting approach for directed energy transport [2]. At medium to high excitation densities, the excitons on the J-aggregates decay mainly due to diffusion driven exciton-exciton annihilation. It is assumed, that multi-exciton states play an important role in the interaction between excitons and in the annihilation process. Although the existence of such states is proven by spectral signatures, population of a multi-exciton state has not been reported yet for an organic system. In this contribution, evidence is provided for significant population of a two-exciton state of PBIl aggregates. Transient absorption spectra are recorded for a wide range of excitation densities and an energy level scheme is presented which explains the observed changes of the spectral signatures in a multi-exciton picture.

\section{Experimental Approach}

Transient absorption measurements are carried out by exciting the sample with $20 \mathrm{fs}$ long pulses at $600 \mathrm{~nm}$ from a noncollinear optical parametric amplifier (NOPA) and using a white light continuum generated in a $\mathrm{CaF}_{2}$-substrate as probe. The transmitted white light is dispersed by a prism and recorded by an array detector. The excitation fluence is varied by a combination of two thin film polarizers. The initial exciton density is estimated from the pump fluence taking saturation effects into account and varies from $3 \%$ per monomer $\left(3.6 \mathrm{~J} / \mathrm{m}^{2}\right)$ to $32 \%\left(57 \mathrm{~J} / \mathrm{m}^{2}\right)$. A methylcyclohexane solution with a concentration of $3 \cdot 10^{-5} \mathrm{M}$ of a substituted PBI1 dye, which forms efficiently Jaggregates [1], serves as sample. 

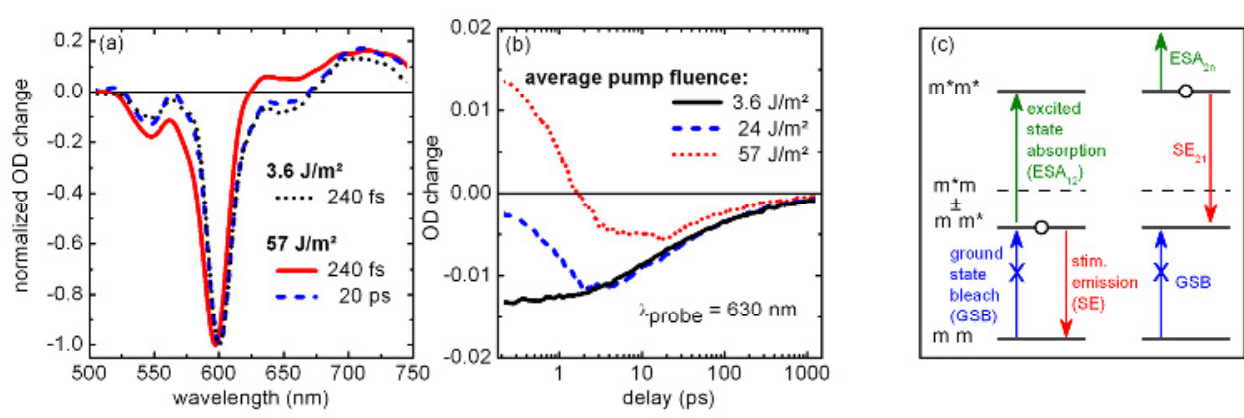

Fig. 1. (a) Normalized transient absorption spectra for low $\left(3.6 \mathrm{~J} / \mathrm{m}^{2}\right)$ and high $\left(57 \mathrm{~J} / \mathrm{m}^{2}\right)$ average pump fluences at two different delay times. (b) Time evolution of the transient absorption at $630 \mathrm{~nm}$ for different average pump fluences. (c) Energy level diagram of the signal contributions for a one- (left) and a two-exciton state (right) in case of a dimer. $\mathrm{m}$ and $\mathrm{m} *$ indicate a monomer in the ground and excited state respectively.

\section{Results}

The transient absorption spectra (Fig. 1a) are dominated by a strong negative feature at about $600 \mathrm{~nm}$ which results from the combination of ground state bleach (GSB), stimulated emission (SE), and excited state absorption (ESA) (Fig. 1c). At low pump fluences (dotted line) the ESA in the region of $600 \mathrm{~nm}$ can be recognized from the blue wing of the negative band which is steeper than the red wing of the SE indicating that the GSB is mostly compensated by the ESA [2]. The ESA results from the transition of the excited one-exciton state into the two-exciton band and is slightly blue shifted compared to the GSB and SE as it is shown in Fig. 1c (left graph) for a dimer model of a Jaggregate. Accordingly, it is not observed in the case of the monomer. Since the bands are broadened by vibrational progressions, strong overlap between ESA and GSB in the aggregates is expected. Another ESA band appears around $700 \mathrm{~nm}$ which is also known from measurements of the monomer and which is attributed to a transition into a higher local electronic state. With increasing pump fluence, the transient optical density around $630 \mathrm{~nm}$ changes from negative to positive for short delay times (Fig. 1a, solid line). This additional absorption is most clearly seen at $240 \mathrm{fs}$ and decays quickly to a negative level which corresponds to the spectra measured at low excitation densities (Fig. $1 \mathrm{~b}$, solid line). We attribute this change to a transient population in the two-exciton manifold. These excitations will lead to the same GSB as one-exciton states but to a blue shifted SE and a slightly red shifted ESA into the three-exciton manifold (Fig. 1c, right graph). Overall, a decrease of the SE and an increase of the ESA contribution in the red wing of the dominant feature should result. This is also in line with the observation that at $240 \mathrm{fs}$ the dominant band shows a significant blue shift at high excitation densities where a large two-exciton population is expected. The effects are not very strong since all the bands are overlapping and the two-exciton population is only a minor part of the generated excitations. The fast decay of the two-exciton manifold can also be seen in the time curve of the transient absorption at $630 \mathrm{~nm}$ (Fig. 1b). Within the first 2 picoseconds the population of the two-exciton states is transferred to the one-exciton manifold and the spectral signatures of the common excitons are completely restored. Due to coherent artifacts the temporal regime below $200 \mathrm{fs}$ is not accessible by our pump probe setup at the moment.

The presented picture also matches the energy dependence and time evolution of excitation density, defined as number of excitons divided by the number of molecules. The excitation density saturates in the case of aggregates at clearly smaller values with increasing pump fluence than for monomers (see Fig. 2a). In aggregates, the excitations are delocalized over several molecules. Accordingly, several monomers are bleached by a single excitation and the saturation threshold is reached at comparatively low pump fluences. It is important to note, that if a two-exciton state is populated and relaxes to the one exciton manifold without splitting into two excitons the number of 
electronic excitations is independent on the fraction of populated two-exciton states. In addition, the oscillator strengths of GSB, SE and ESA into higher exciton manifolds are ideally equal and the integrated signal strength, which is used to calculate the exciton density, varies only little between a bright one- and a two-exciton state. For these reasons, the saturation behavior is little influenced by the population of two-exciton states. However, it is obvious that at high pump fluences the density of electronic excitations is strongly saturated. In this case the probability for absorbing a second photon and exciting into the two-exciton manifold is quite high.
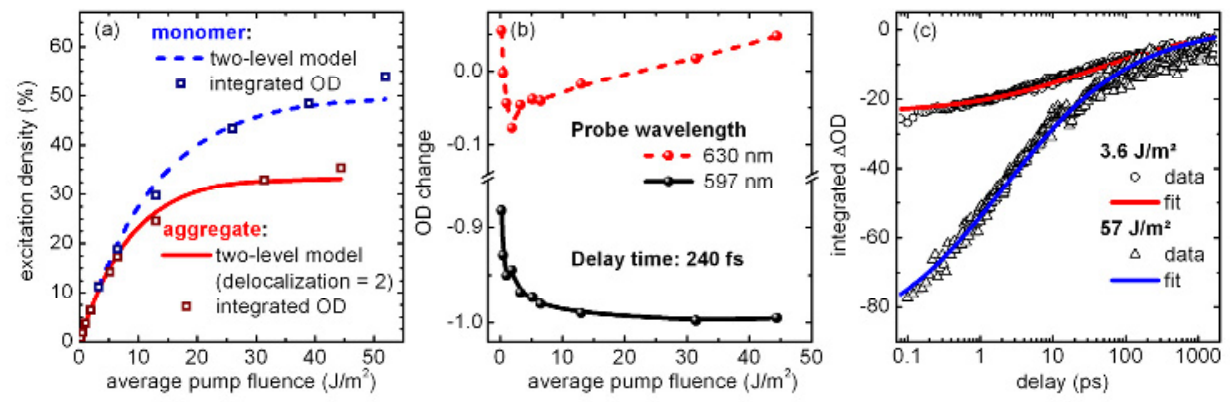

Fig. 2. (a) Excitation density in monomer units for PBI1 aggregates (solid line) and PBI1 monomers (dotted line, in dichloromethane). (b) Transient absorption of PBI1 aggregates in dependence of the pump fluence for two different wavelengths. (c) Time evolution of the integrated transient spectra for a low $\left(3.6 \mathrm{~J} / \mathrm{m}^{2}\right.$, circles) and a high $\left(57 \mathrm{~J} / \mathrm{m}^{2}\right.$, triangles) pump fluence. The solid lines are fits of a diffusion driven annihilation model.

The increasing population of the two-exciton state at rising pump fluences can also be seen in Figure $2 \mathrm{~b}$. The transient absorption at $597 \mathrm{~nm}$, which can mainly be attributed to the single exciton species, saturates at relatively low pump fluences. In contrast, the transient absorption at $630 \mathrm{~nm}$ increases at higher fluences linearly with increasing pump fluence. In comparison to the aggregate saturation curve (Fig 2a, solid line) this linear increase proves the population of a higher excited state, which is visible at this particular wavelength, by two photon absorption.

The dynamics on longer time scales are dominated by exciton decay due to diffusion driven annihilation as it is described in [2]. This is demonstrated in Fig. 2b by the good agreement between the signal evolution (symbols) and the annihilation model (solid lines). It indicates that excitons generated via a transient population of the two-exciton manifold behave in the same way as excitons generated by absorption of a single photon. However, it is not yet clarified why no significant deviations from the simple annihilation model are observed at short delay times and if the mobility of an excitation in the two-exciton manifold is similar to that of a common exciton.

In summary, transient absorption spectroscopy is performed in an intensity regime where a significant excitation of the two-exciton manifold is expected. The observed spectral changes compared to low pump energies provide indeed evidence that this population exists and relaxes to the one-exciton manifold within 2 ps. The lifetime of the two-exciton state is surprisingly long especially when compared to the relaxation dynamics of the exciton-exciton annihilation process that should be significantly faster [2]. Consequently, the two-exciton state is most probably not involved in the annihilation process.

\section{References}

1. X.-Q. Li, X. Zhang, S. Ghosh and F. Würthner, Chem. Eur. J. 14, 8074 (2008).

2. H. Marciniak, X.-Q. Li, F. Würthner and S. Lochbrunner, J. Phys. Chem. A 115, 648 (2011). 